\title{
Introduction to IT Architectures and Implementations in Healthcare Environments Minitrack
}

\author{
Radmila Juric \\ USN, Norway \\ rju@usn.no
}

\author{
Karen Stendal \\ USN Norway \\ Karen.Stendal@,usv.no
}

\author{
Sang Suh \\ Texas A\&M University- \\ Commerce, US \\ Sang.Suh@tamuc.edu
}

This year we selected four papers, which fit under the umbrella of applications and architectures for IT in healthcare environments. They are very different and belong to a selection of problem domains in healthcare where software technologies either support or enable the efficiency of healthcare deliveries.

The paper Influencing Factors of Clinical Patient Recruitment Systems Design elaborates on the on the holistic design for clinical patient recruitment systems, for the purpose of defining critical success factors of clinical trial performances. The authors defined these factors, and found new ones, through extensive literature review and several semistructured expert interviews. The outcome is interesting: their classification and systematization of influence factors for clinical patient recruitment systems, could be a foundation for specifying requirements for modelling clinical patient recruitment systems in future.

The paper A multi-sensor system for enhancing situational awareness and stress management for people with ASD in the workplace and in everyday life, addresses a very important set of challenges autistic people face in their everyday lives. The paper illustrates the architecture and deployment of the project, which focuses on the positive behaviour support, within a customised environment, aiming to minimise negative impacts of deviations in factors like lighting or sound to the stress level of autistic people. The outcome is a framework, which allows for constant monitoring of the environment and combining information from different sensor channels, including personal and environmental sources. The architectural model is deployable as an application within mobile and wireless operating environments.
The paper Towards an Artificial Pancreas: Software Architectural Model and Implementation for Personalized Insulin Administration draws attention to the research field of developing an Artificial Pancreas and illustrates numerous problems related to data collection and their computations, which are supposed to mimic the functioning and performance of the natural organ. The proposed software architectural model shows a new set of data and computations which address deficiencies of current insulation infusion pumps, used by millions of diabetic patient all over the world. It also shows that an excerpt of the proposed architecture, deployed using Watson Analytics and performed upon a selection of data collected from sensors, individual patient's input and persistent patient records, are far more powerful in understanding how to address insulin infusion, than any of the current solutions we have in the market and research laboratories. A shift in thinking and transdisciplinary research are needed to address the deficiency of insulin infusion pumps and computational models, which take into account only a very limited number of factors when calculating insulin infusion levels.

The paper A Case Study Perspective to the Digital Transformation of a Hospital's Perioperative Process comes from our long-term contributors who explored implementations of software technologies across large hospital environments for the last 18 years. In this submission, they look at a case study, which identifies existing perioperative sub-process limitations, potential capabilities, and subsequent sub-process contextual understanding to minimize perioperative process complexity. They discovered that specific perioperative nursing documentation, plus electronic medical records, demonstrate the utility and value of patient-centric perioperative data. Consequently the data plays the role of organizational resource for process management and control. The paper also discusses, theoretical and practical implications of the case study. 